\title{
Monitoring of spatial data coordinate basis integrity using coordinate transformations
}

\author{
Vicktor Nepoklonov ${ }^{1 *}$, Mayya Maximova ${ }^{1}$, and Ivan Sukharev-Krylov ${ }^{1}$ \\ ${ }^{1}$ Moscow State University of Geodesy and Cartography, Moscow, Russia
}

\begin{abstract}
The modern spatial data coordinate basis (SDCB) is built taking into account the variety of existing and used today geodetic networks, models of physical fields of the Earth, cartographic models, as well as coordinate systems (CS). One of the requirements for SDCB from the standpoint of system analysis is the requirement of integrity, which presupposes the unity of the determination of coordinates, that is, the consistency of the results of determining the coordinates of the same points in different CSs. The article is devoted to the monitoring of the accuracy characteristics of the available software for coordinate transformations in terms of single-stage and multi-stage transitions between ellipsoidal coordinates of different systems.
\end{abstract}

Keywords: coordinate basis, spatial data, coordinates transformations, iterative methods, direct methods, transformation accuracy

\section{Introduction}

The practice of working with spatial data indicates a growing variety of CSs [1] used. If to consider conventionally one of these CS as basic one, then the integrity criterion can be the proximity of the same-name coordinates of points in this system, obtained directly and by mathematical transformation from other CSs to the basic CS.

Consequently, one of the essential factors for ensuring the integrity of the SDCB is the accuracy of models, methods and algorithms for transforming coordinates, both in theory and in terms of their implementation in software, and the tasks of SDCB monitoring naturally include the tasks of monitoring the accuracy characteristics of the available mathematical and software coordinate transformations.

Typical tasks of transforming coordinates in their variety are schematically shown in Figure 1.

\footnotetext{
* Corresponding author: vbnep@mail.ru
} 


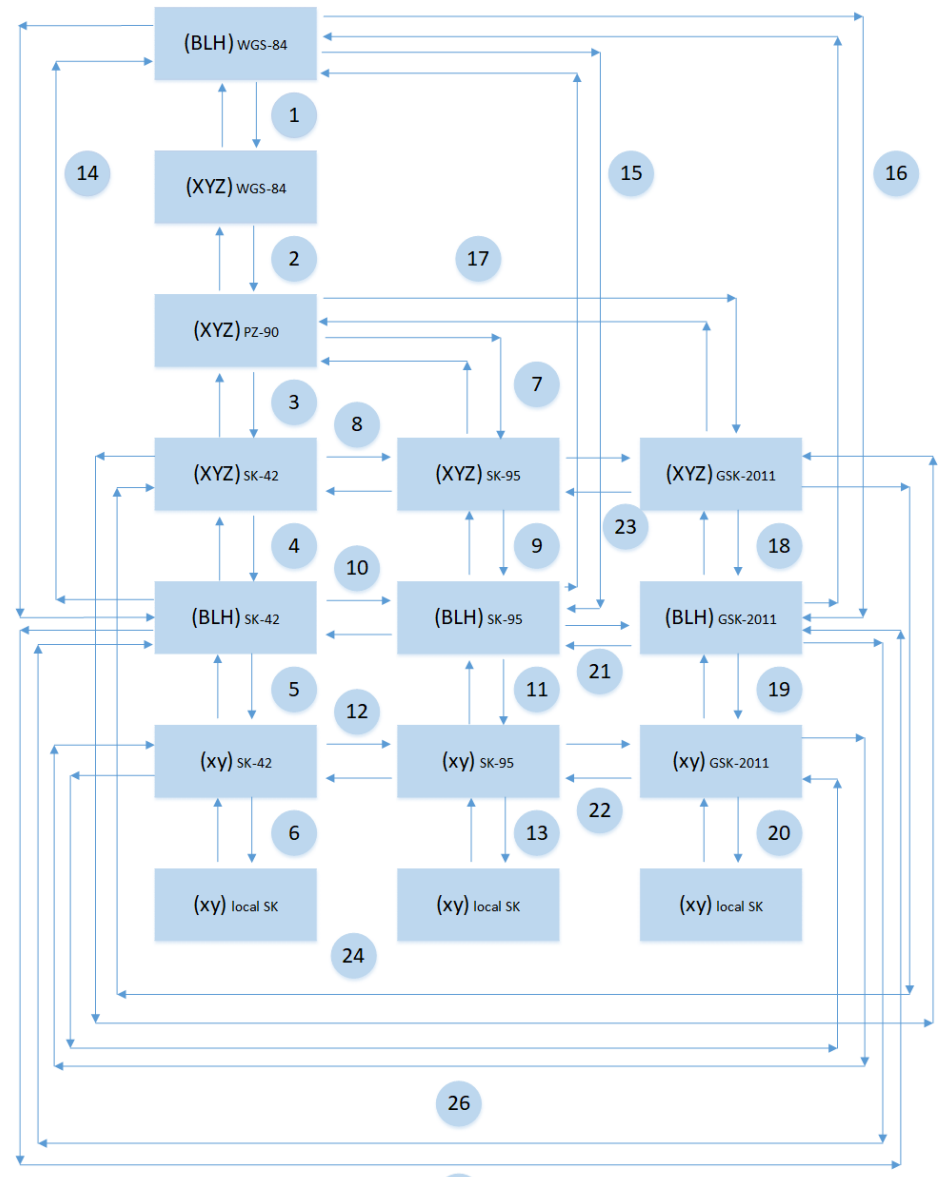

25

Fig. 1. Scheme of typical tasks of transforming coordinates.

One of the main tasks is transformation is presented in formula (1):

$$
\{B, L, H\}_{1} \rightleftarrows\{B, L, H\}_{2}
$$

and its special case - formula (2):

$$
\{B, L\}_{1} \rightleftarrows\{B, L\}_{2}
$$

where $B, L, H$ - geodetic (ellipsoidal) coordinates (latitude, longitude, height); «1»- first coordinates system; «2» $\quad$ - $\quad$ second coordinates system; «६»- pair of transformations «forward - backward».

Foreign and domestic scientists: M. Bursa [2], W. Heiskanen, H. Moritz [3], J. Badekas [4], B. Bowring [5], K. Borkowski [6], B. Hofmann-Wellenhof [7], T. Fukushima [8], R. Deakin [9], W. Featherstone [10], H Vermeille [11], M. Molodensky [12], L. Pellinen [13], V. Morozov [14], M. Yurkina [15], Yu. Komarovsky [16], V. Balandin, M. Bryn, A. Matveev [17], L. Ogorodova [18], V. Krylov [19], K. Afonin [20] et al made a significant contribution to the formation and development of research on these issues. 
Monitoring of the methodological approaches used to solve this task shows that two approaches are currently being used.

One of them involves performing the transformation «1» directly, i. e. using differential formulas of spheroidal geodesy, given, in particular, in [18-20].

The second approach is based on the use of a multi-stage scheme $\{B, L, H\}_{1} \rightarrow\{X, Y, Z\}_{1} \rightarrow\{X, Y, Z\}_{2} \rightarrow\{B, L, H\}_{2}$

At the first step, spatial rectangular coordinates are calculated using the formulas of spheroidal geodesy [12-14]. The second transformation is performed by Helmert's formulas $[7,16,18,19]$. An alternative is to use the Molodensky-Badekas formula $[4,9,16]$. At the third step, the longitude $L_{2}$ is obtained according to the well-known formula $[3,7,13,19]$ and various methods can be used to calculate the latitude $B_{2}$ and the height $\mathrm{H}_{2}$, each of them has its own advantages and disadvantages.

According to the applied mathematical apparatus, these methods can be divided into two groups:

Iterative methods

- Heiskanen and Moritz's Method (1967);

- Bowring's Method (1976);

- $\quad$ Lin and Wang's Method (1995);

- Jones' Method (2002);

- $\quad$ Pollard's Iterative Method (2002);

- Barteleme's Method (2003)

- GOST R 51794 (2017) et al.

Non-iterative methods

- Paul's Method (1973);

- Ozone's Method (1985);

- $\quad$ Borkowski's Method (1989);

- Vermeille's Method) (2002);

- $\quad$ Sunkel's Method (1998);

- $\quad$ Shu's Method (1993) et al.

Iterative methods, in turn, can be divided into two more groups depending on the calculation of the height: those methods where the height is determined by iterating sequentially together with the iterative calculation of latitude, and methods where the height is determined at the end of the calculation after the completion of the latitude iterations.

\section{Materials and methods}

With increased requirements to the accuracy of SDCB implementation in the part of coordinate transformations, it is important to know to what extent the results of transforming the coordinates of the points being determined, obtained using various methods, are consistent with each other. For this purpose, in this work, a comparative study of the two methodological approaches described above was carried out using the example of converting coordinates from the WGS-84 system (system 1) to the SK-42 coordinate system (system 2).

This study was carried out as part of a computational experiment for comparing various methods of coordinate transformation.

To carry out this experiment, it is necessary to make a special computational program that sufficiently takes into account the features of the problem under study.

Based on these features, the specified program and the methodology for conducting experimental research implemented in it should provide: 
- $\quad$ setting the coordinates of calculated points in various limits, from a separate area to the globe as a whole, and with different discreteness in the planning aspect (from several degrees or more to several arc minutes) and in height (from several tens to several hundred meters or more);

- variation of the initial and final coordinate systems and, accordingly, variation of the elements of mutual transformations of coordinates and parameters of the reference ellipsoids;

- implementation of two different coordinate transformation schemes - straight line and stepped, as well as the implementation of various methods for recalculating geodetic latitudes and heights, both iterative and non-iterative;

- formation of control points array, recalculation of their coordinates from the original system (system 1) to the final coordinate system (system 2) using various patterns and methods, assessment and analysis of the resulting discrepancies in coordinates values of the like points in system 2 (in numerical and graphic types);

- control assessment of the accuracy of alternative patterns and methods of coordinate transformation by comparing the initial values of the coordinates of the determined points in system 1 with their restored values obtained by inverse transformation from system 2 with the same transition parameters (in numerical and graphic forms).

These requirements are met by a computational program developed by the authors in Python 3.8, which was used in this work to perform experimental calculations in the framework of a comparative study of various patterns and methods of coordinate transformation. It should be noted that the transition from the WGS-84 system to the SK-42 system is implemented in this program in accordance with GOST R 51794-2017 (that is, via the PZ-90.11 intermediate system).

One of the main blocks of the program is the module for conversion from rectangular coordinates to ellipsoidal coordinates using various methods described above. Its prototype was the state-registered CoordTransformer program developed with the participation of the authors [21]. Plotting and visualization of graphs is provided by the MatPlotLib library tools imported by the program, which is freely distributed for the Python language. In addition to this, the graphical tools of the Microsoft Office Excel program were used.

\section{Results and discussion}

For the experiment, the calculations were performed with the following initial data: latitude range - from $-90^{\circ}$ to $+90^{\circ}$, pitch $-5^{\circ}$ (within a radius of 30 'relative to the poles $-1^{\prime}$ ); longitude range - from $0^{\circ}$ to $360^{\circ}$, pitch $-90^{\circ}$; range of geodetic height change - from 0 to $15 \mathrm{~km}$, step - $100 \mathrm{~m}$; the parameters of the reference ellipsoids WGS-84 and SK-42 are taken according to GOST R 51794-2017.

It should also be noted that when considering the accuracy of calculating ellipsoidal coordinates, emphasis will be made on the accuracy of calculating ellipsoidal latitude. This is due to the fact that the ellipsoidal longitude for all multistage transition methods can be determined using only one formula (without taking into account the transition to a semicircular counting). Therefore, the calculation of longitude is assumed as equidistant for any method of calculation, and the ellipsoidal height in the multistage transition is most often a function of latitude. Therefore, the more accurately the ellipsoidal latitude is calculated, the more accurately the height will be calculated.

Let's consider the graphs of transformation accuracy for a single-stage transition between ellipsoidal coordinates from WGS-84 to SK-42 and back to WGS-84 for circumpolar latitudes and latitudes outside the poles. 
As mentioned above, the longitude search pitch for circumpolar latitudes was $90^{\circ}$. The accuracy graphs of Southern and Northern circumpolar latitudes for longitude $0^{\circ}$ are shown in Figure 2. No other values of ellipsoidal longitude are considered here, since the value of longitude does not significantly affect the accuracy of calculations of ellipsoidal latitude.

$\mathrm{dB}, \mathrm{B}= \pm 90$

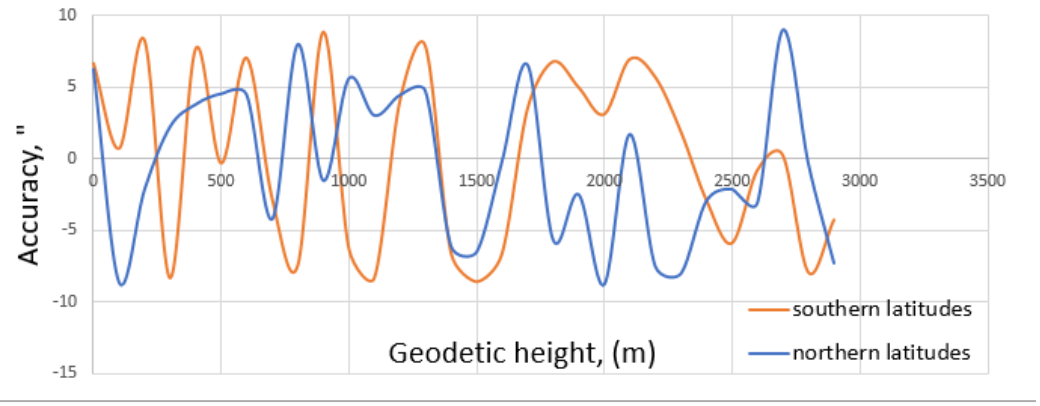

$\mathrm{dB}, \mathrm{B}=-89.9 \ldots-89.5$

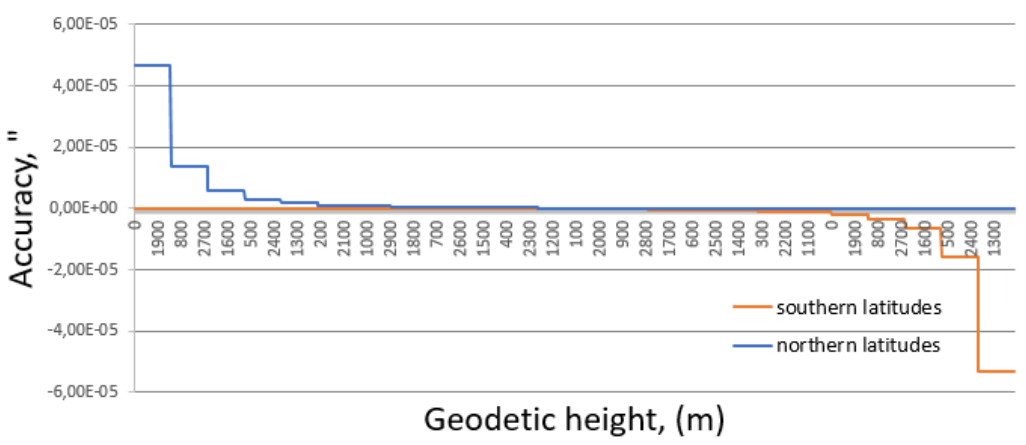

Fig. 2. Accuracy of calculations for circumpolar latitudes (single-stage transition).

The accuracy graph of single-stage transition transformation for ellipsoidal latitudes within the range from -89 to $89^{\circ}$ with a $5^{\circ}$ pitch is shown in Figure 3 . The longitude in the given graph is equal to zero, and the heights are in the range of $0 \ldots 15000 \mathrm{~m}$ with a pitch of $100 \mathrm{~m}$. 


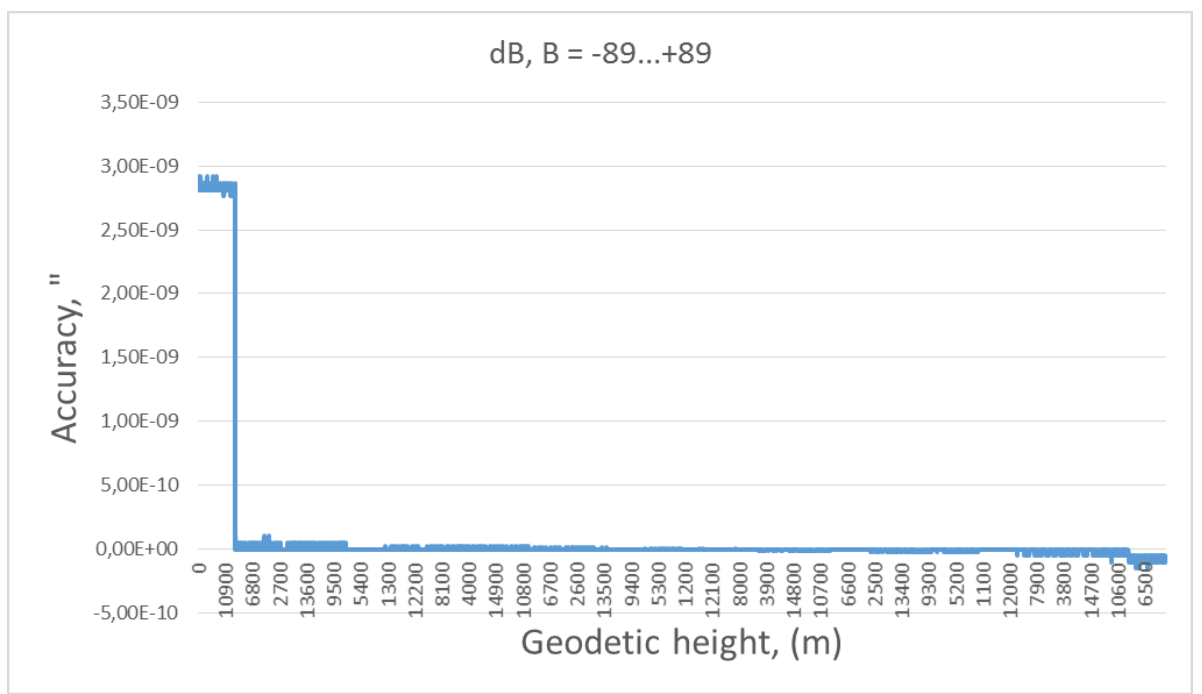

Fig. 3. Accuracy of calculations for non circumpolar latitudes (single-stage transition).

Let's consider a multistage method of transition between ellipsoidal coordinates, where transformations at the last stage are performed using direct and iterative methods.

A single-stage transition example showed that a change of geodetic longitude does not significantly affect the accuracy of calculation of geodetic latitude - none of the values considered above changed by an order of magnitude with a significant change (with a pitch of 120 degrees) of geodetic longitude. Therefore, in order to reduce the amount of calculations, and to comply with the optimal number of graphs, the multistage method of transition between curved ellipsoidal coordinates will be considered only for the selected value of geodetic longitude equal to $120^{\circ}$.

Figures 4-5 show the graphs of accuracy of latitudes calculation by a multistage transition near the South Pole using some of the transformation methods we studied (both direct and iterative), with a pitch of 1' in latitude and $100 \mathrm{~m}$ in height. At that, in southern latitudes, it is not possible to make calculations by Ozone and Paul methods.

Figure 6 shows, as an example, graphs of calculation accuracy when using the methods of Jones, Lin and Wang, Pollard and Sunkel for latitudes near the North Pole. 
$\mathrm{dB}, \mathrm{B}=-90 \ldots-89.5$

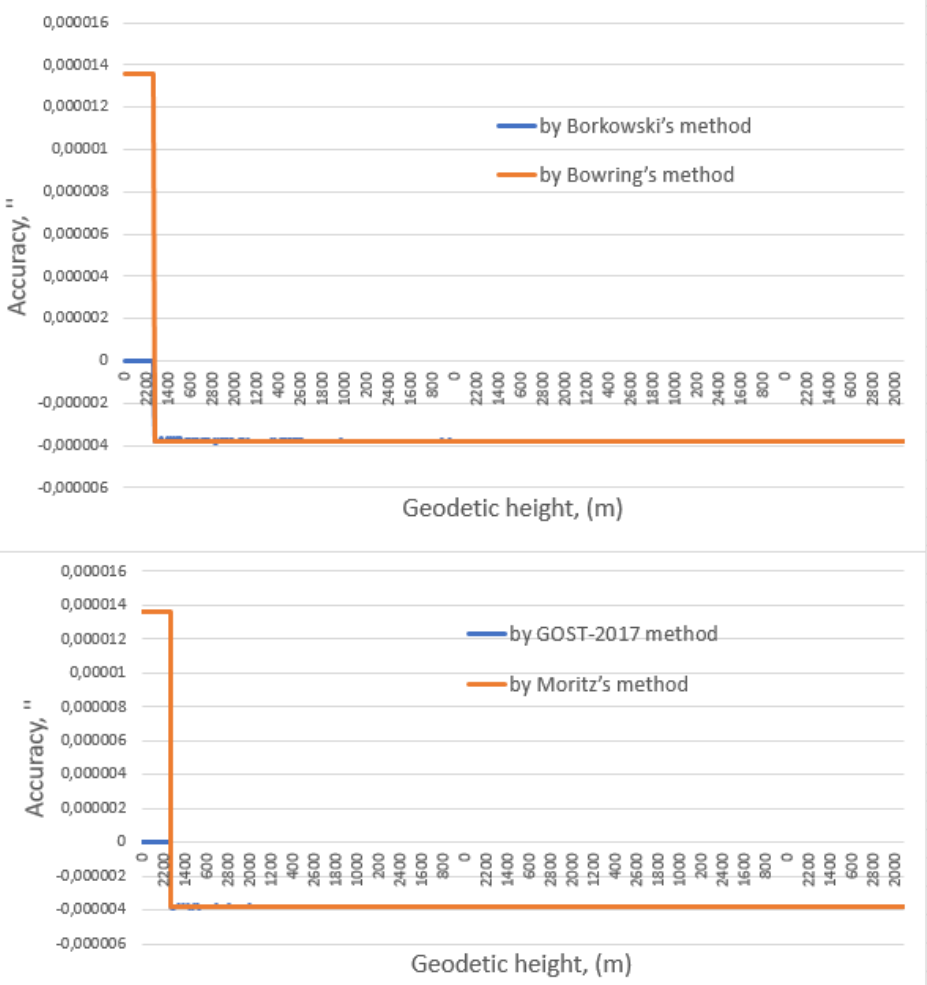

Fig. 4. Accuracy of calculations when using the methods of Bowring, Borkowski, Helmut-Moritz and GOST-2017 for latitudes near the South Pole (multistage transition).
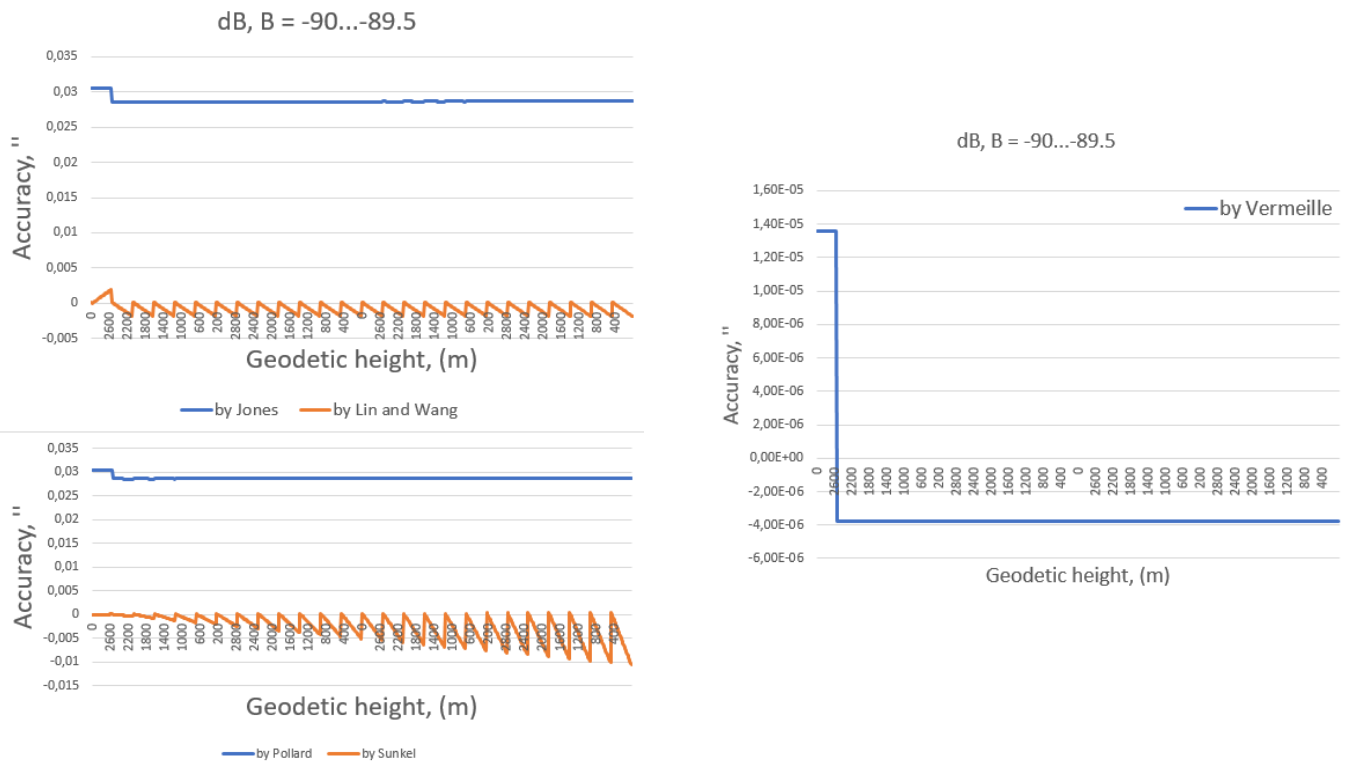

Fig. 5. Accuracy of calculations when using the methods of Jones, Lin and Wang, Pollard, Sunkel and Vermeille's method for latitudes near the South Pole (multistage transition). 


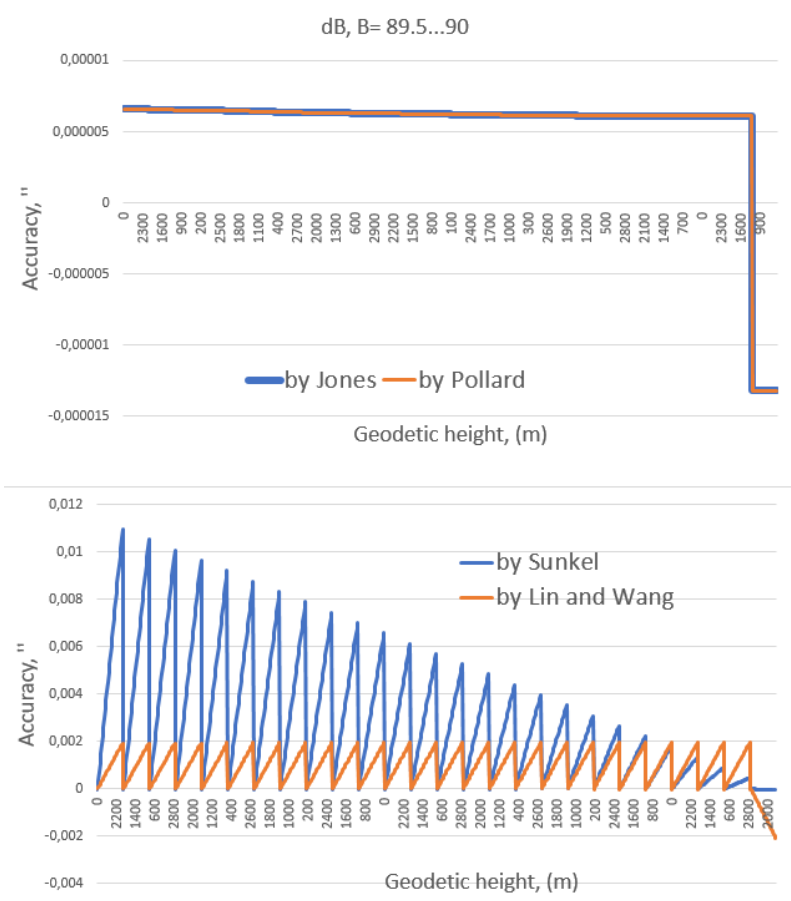

Fig. 6. Accuracy of calculations when using the methods of Jones, Lin and Wang, Pollard and Sunkel for latitudes near the North Pole (multistage transition).

Figures 7-8 show the graphs of calculation accuracy for the multistage transition using Moritz and Pollard methods, built using the matplotlib library for latitudes outside the poles.

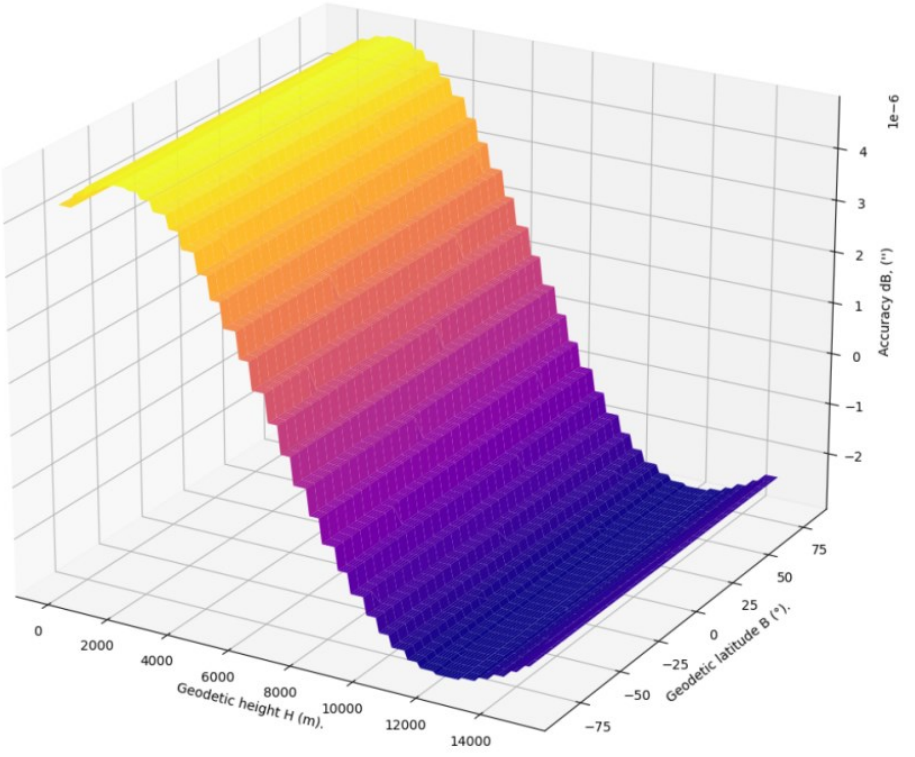

Fig. 7. Accuracy of calculations when using Moritz method for latitudes outside the poles (multistage transition). 


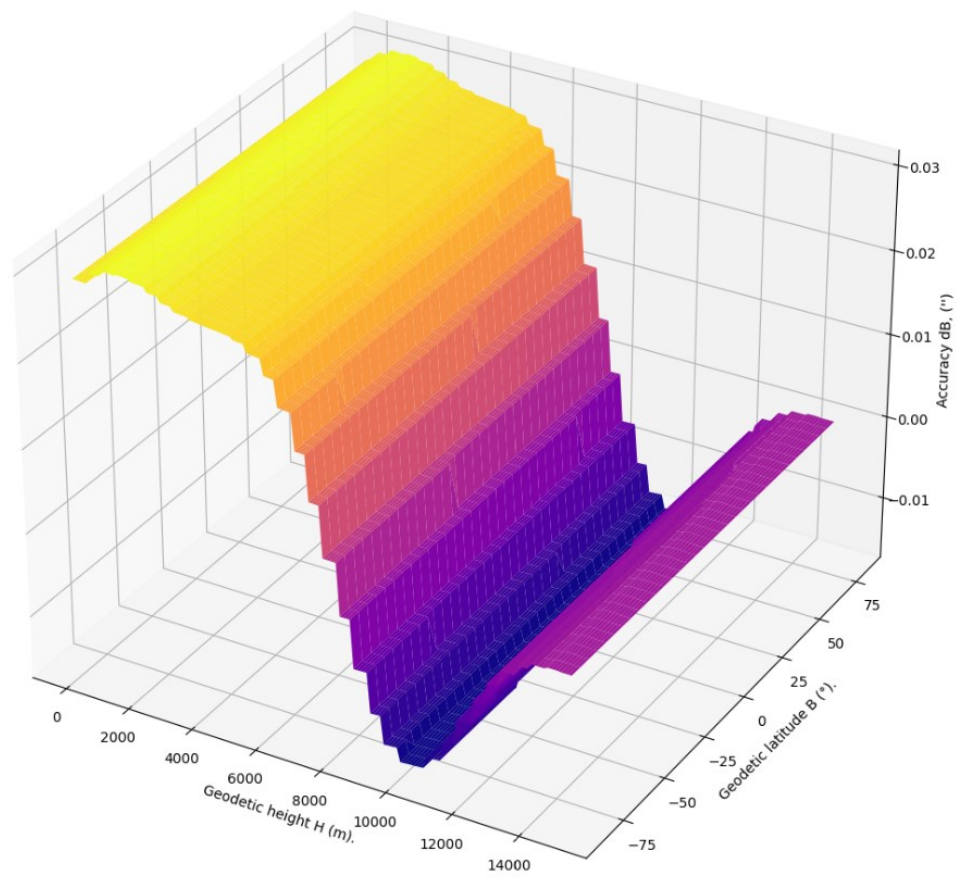

Fig. 8. Accuracy of calculations when using Pollard method for latitudes outside the poles (multistage transition).

In addition to the graphs, the average values of accuracy of calculation of ellipsoidal latitude in all the subject ranges, and root-mean-square errors (RMS) of calculating the ellipsoidal latitude, which were calculated separately for circumpolar latitudes and noncircumpolar ones, were also used as additional analysis criteria.

\section{Conclusion}

In this work, a comparative study of accuracy characteristics of the main coordinate transformations, which can be used to monitor the integrity of SDCB, was carried out. The study showed that when choosing a method of coordinate transformations, it is necessary to take into account the requirements for the integrity of SDCB in various applications, and when the requirements for accuracy of coordinate system harmonization are higher, the geographic factor shall be taken into account too.

It is deduced from experiments that near the poles of the Earth, a single-step transition between the ellipsoidal coordinates of different Coordinate Systems gives a rather large error (about 10 arcsec). It is preferable to use the methods (algorithms) of a multistage transition, while, according to our evaluations, the most accurate way to calculate ellipsoidal coordinates at the last stage is the Vermeille method, which provides an average determination of coordinates with an error of $5.3 \times 10^{-6}$ arcsec. Outside the Polar Regions, it is more expedient to use a single-stage transition procedure, which showed an average accuracy of $2.9 \times 10^{-10}$ arcsec over the entire range of heights, latitudes and longitudes. This is about four orders of magnitude less compared to the same accuracy characteristics of a multistage transition. 
Thus, the currently available mathematical tool for coordinate transformations allows to monitor SDCB almost without restrictions on the accuracy of the geographic location of the subject areas.

\section{References}

1. V.B. Nepoklonov, M.V. Maximova, Izvestia Vuzov. Geod. and Aerophotosurv. J., 1, 22-28 (2016)

2. M. Bursa, Studia Geophysica et Geodetica, 6, 209-214 (1962)

3. W. A. Heiskanen, H. Moritz, Physical geodesy, 374 (1967)

4. J. Badekas, Investigations related to the establishment of a world geodetic system, Report No. 124, Department of Geodetic Science, Ohio State University, Columbus, Ohio (1969)

5. B. Bowring, Surv. Review J., 23 (181), 323-327 (1976)

6. K. Borkowski, Bull. Géod. J., 63, 50-56 (1989)

7. B. Hofmann-Wellenhof, H. Lichtenegger, J. Collins, Global Positioning System: Theory and Practice, 313 (1992)

8. T. Fukushima, J. of Geodesy, 12 (79), 689-693 (2006)

9. R.E. Deakin, A note on the Bursa-Wolf and Molodensky-Badekas transformations, 21 (2006)

10. W.E. Featherstone, S.J. Claessens, Geophys. Geod. J., 52, 1-18 (2008)

11. H. Vermeille, J.of geodesy, 85, 105-117 (2011)

12. M.S. Molodensky, V.F. Yeremeev, M.I. Yurkina, Methods of studying the external gravitational field of the Earth and the figure of the Earth, in Proceedings of the TsNIIGAiK, Moscow (1960)

13. L.P. Pellinen, Higher geodesy: Theoretical geodesy, 264 (1978)

14. V.P. Morozov, Spherical geodesy, 2, 296 (1979)

15. M.I. Yurkina, L.I. Serebryakova, Izvestia Vuzov. Geod. and Aerophotosurv. J., 3, 4053 (2001)

16. Yu. A. Komarovsky, The use of various datum-ellipsoids in navigation, 341 (2005)

17. V.N. Balandin, M.Ya. Bryn, A.Yu. Matveev [et al], Geodesy and Cartography J., 6, 15-16 (2006)

18. L.V. Ogorodova, Izvestia Vuzov. Geod. and Aerophotosurv. J., 2, 63-66 (2014)

19. V.I. Krylov, Coordinate-time transformations in geodesy: tutorial, 114 (2014)

20. K.F. Afonin, Interexpo Geo-Siberia J., 1 (1), 160-163 (2015)

21. M.V. Maximova, I.A. Sukharev-Krylov, Rospatent, Computer Program State Registration Certificate №2019662879 «CoordTransformer» dated 04 October (2019) 